\title{
Einleitung und Anweisung zum Gebrauch der Tabellen.
}

$\mathrm{U}^{\mathrm{m}}$ einen verständnisvollen Gebrauch der Tabellen zu ermöglichen, gebe ich im folgenden die Berechnungsweise derselben kurz an.

Es wurden zunächst die Widerstandshöhen in Millimeter Wassersäule für eine große Anzahl von Kesseln verschiedenster Konstruktion und Gliederzahl bei normaler Beanspruchung berechnet. Ebenso wurden die Widerstandshöhen für cine Anzahl von Radiatoren verschiedenster Bauhöhe und Gliederzahl bestimmt. Als Vorlauftemperatur des Heizwassers wurden $+90^{\circ} \mathrm{C}$, als Rücklauftemperatur $+60^{\circ} \mathrm{C}$ angenommen. ${ }^{1)}$

Diese Temperaturdifferenz wurde von mir deshalb gewählt, weil sie in allen mir vorgekommenen Fällen sich stets in der Praxis gut bewährt hat, und ich daher keinen Grund dafür einsah, mit einer geringeren Temperaturdifferenz zu rechnen, die - selbst bei nur etwas umfangreicheren Anlagen - bedeutend größere Rohrweiten und damit höhere Anlagekosten ergibt, die ohne Nachteil für die Funktion der Anlage vermieden werden können. Die sich ergebende mittlere Oberflächentemperatur der Heizkörper von ca. $+75^{\circ} \mathrm{C}$ dürfte als keine zu hohe angesehen werden, namentlich da sie ja nur vorübergehend bei scharfer Kälte erreicht wird.

1) Siehe Bemerkung an Schiluß der Tabellen.
Durch die Bestimmung der Widerstandshöhen verschiedener Kessel und Radiatoren kam ich empirisch zu dem Resultat, daB man, ohne einen bedeutenden Fehler zu machen, den Summen der Widerstandshöhen für Kessel und Radiatoren je nach dem Umfange der Anlage die Werte von 2,5 bis 10,0 beilegen kann unter der Voraussetzung, dab die Gliederzahl der Radiatoren etwa 20 Glieder beträgt und der Anschluß der Kessel sowohl wie der Radiatoren einseitig erfolgt.

Wie die Tabellen erkennen lassen, wurde als kleinste Anlage diejenige behandelt, deren entferntester Strang in einer horizontalen Enfernung vom Kessel von 5,0 m liegt, als größte Anlage diejenige, deren entferntester Strang in einer horizontalen Entfernung von $60,0 \mathrm{~m}$ vom Kessel liegt. Die Berechnung erfolgte nun unter der Annahme einer gleichmäßigen Verteilung des Druckverlustes auf den Stromkreis, welcher durch den am ungünstigsten gelegenen Heizkörper geht, d. h. durch den Heizkörper, der sich im ErdgeschoB am entferntesten Strange befindet. In Abständen von $5 \mathrm{zu} 5 \mathrm{~m}$ wurde die Abzweigung eines Stranges von dieser horizontalen Verteilungsleitung angenommen und die bis $z u$ diesen Abzweigpunkten verbrauchten Druckhöhen berechnet. Durch Abrechnung dieser und der für Kessel und Radiatoren angenommenen Widerstandshöhe von 
der erreichbaren Druckhőhe der einzelnen Heizkörper des Erdgeschosses wurde die noch zur Verfügung stehende Druckhöhe für die letztere ermittelt. Die in der Praxis meist zutreffende Annahme, daB die vertikale $\mathrm{Zu}$ - und Rückleitung der Heizkörper des Erdgeschosses eine Länge von $2,0 \mathrm{~m}$, die horizontale eine Länge von $3,0 \mathrm{~m}$ besitzt, gestattete alsdann die Bestimmung der pro laufenden Meter zur Verfügung stehenden Druckhöhe.

Die Berechnung der Leitungen nach und von den Heizkörpern des I. Obergeschosses erfolgte analog der im Vorhergehenden angegebenen Berechnungen für die Heizkörper des Erdgeschosses, immer naturgemă $B$ unter Abzug der bereits verbrauchten Druckhöhen.

Als Geschoßhöhe wurden hierbei $3,5 \mathrm{~m}$ angenommen. Ist dieselbe größer, so wird doch eine bedeutendere Differenz der pro Ifd. Meter zur Verfügung stehenden Druckhöhe sich deshalb nicht ergeben, weil alsdann bei Bestimmung derselben nicht allein die verfügbare Druckhöhe, welche als Zăhler auftritt, anwächst, sondern auch die Lănge der Strecke, die den Nenner des Bruches bei Vornahme der Rechnung bildet.

Die Leitungen zu und von den Heizkörpern der höher gelegenen Geschosse wurden genau in derselben Weise wie diejenigen ermittelt, welche zu den Heizkörpern des I. Obergeschosses führten. Die Annahme einer GeschoBhöhe von $3,5 \mathrm{~m}$ und der Länge von $3,0 \mathrm{~m}$ für die Heizkörperanschlüsse wurde auch hierbei beibehalten.

Auf die soeben beschriebene Weise entstand der Kopf (\$) I. Horizontale Leitung () und die linke Hälfte der unter " II. Vertikale Strănge» aufgeführten Tabellen, bei welcher — wie die Überschrift angibt - die Voraussetzung gemacht wurde, daB sich Heizkörper im ErdgeschoB und in den Obergeschossen befinden.

Für den Fall, daß ein Strang - wie dieses bei Hăusern, die im Erdgeschoß Geschäftsräume und in den Obergeschossen Wohnräume enthalten, hăufig vorkommt - nur in den Obergeschossen Heizkörper besitzt, ist die rechte Hălfte der Tabellen errechnet worden, da sich alsdann die Rohrdimensionen teilweise verschieben.

Der im Vorstehenden angegebene Entstehungsgang der Tabellen dürfte im allgemeinen zur sachgemäßen Anwendung derselben genügen. Wenn im folgenden dennoch einige erläuternde Worte hierüber hinzugesetzt werden, so geschieht dieses, um irrtümliche Anwendungen nach Möglichkeit von vornherein auszuschließen, auch sollen noch einige Hinweise darüber angegeben werden, wie in einzelnen "Grenzfällen « $z u$ verfahren ist.

Zunăchst ist die Entfernung zwischen der Mitte des untersten Heizkörpers im ErdgeschoB und der Mitte des Kessels sowie die horizontale Entfernung des letzten Stranges vom Kessel zu bestimmen. Hiernach erfolgt die Auswahl der Tabelle.

Nachdem an allen Teilstrecken die durch dieselben gehenden Wärmeeinheiten vermerkt sind, erfolgt zunächst die Dimensionierung der horizontalen Verteilungsleitung nach dem hierfür errechneten Kopf jeder Tabelle (I. Horizontale Leitung). Teilt sich die Leitung am Kessel oder in nicht allzu weiter Entfernung von demselben in zwei oder mehrere ungleich lange Äste, so sind für die Bestimmung der Rohrweiten jedes dieser Äste verschiedene Tabellen zu wählen. Die gemeinsame Anschlußleitung am Kessel ist hierbei naturgemäß nach der Tabelle für den längsten Ast zu bestimmen. Kurze horizontale Verbindungen - bis zu höchstens etwa $5,0 \mathrm{~m}$ Länge - zwischen Strang und Verteilungs- 
leitung können wie die Dimensionen des Stranges ausgeführt werden.

Zur Ermittlung der Rohrweiten der einzelnen Stränge ist es erforderlich, die horizontale Entfernung derselben vom Kessel zu bestimmen. Es empfiehlt sich, diese in der Strangzeichnung unter der Nummer jeden Stranges zu vermerken. Befindet sich auch im Erdgeschoß ein Heizkörper am Strang, so erfolgt die Dimensionierung des ganzen Stranges nach der linken Hälfte der Tabelle für vertikale Stränge, andernfalls nach der rechten Hälfte. Am besten bestiınmt man die Rohrweiten und die Heizkörperanschlüsse hintereinander von oben herab für jeden Strang. Hierbei kann es - namentlich in den oberen Geschossen vorkommen, daß nach der Tabelle z. B. die Anschlüsse für zwei kleine Heizkörper im obersten Geschoß eine lichte Weite von $13 \mathrm{~mm}$ erhalten, und daß der Steigestrang zu ihnen auch nur $13 \mathrm{~mm}$ stark zu werden braucht. Dieses hat darin seinen Grund, daß die Tabelle nicht die Werte für Anschlüsse von $10 \mathrm{~mm}$ lichter Weite enthălt. In solchem Falle ist dann, wenn der Steigestrang nur noch gerade mit $13 \mathrm{~mm}$ genügen würde, die Zuleitung desselben $20 \mathrm{~mm}$, die Rückleitung $13 \mathrm{~mm}$ weit $\mathrm{zu}$ machen. Die oberen Anschlüsse der Heizkörper sind $13 \mathrm{~mm}$, ihre unteren Anschlüsse $10 \mathrm{~mm}$ weit anzulegen.

Ähnlich ist zu verfahren, wenn einer oder beide Anschlüsse etwa eine lichte Weite von $20 \mathrm{~mm}$ nach der Tabelle erhalten müßten und der Strang gleichfalls noch mit $20 \mathrm{~mm} \mathrm{l.} \mathrm{Durchm.}$ genügte. Eine Verstärkung des Stranges ist in diesem Falle nicht immer nötig, vielfach genügt es, wenn die unteren Heizkörperanschlüsse dann $13 \mathrm{~mm}$ werden, oder auch wenn man den oberen Anschlüssen und den Ventilen eine lichte Weite von nur $13 \mathrm{~mm}$ gibt und dafür die unteren Anschlüsse der Heizkörper $20 \mathrm{~mm}$ weit macht. Letzteres empfiehlt sich dann, wenn die Heizkörper nur wenig mehr Wărmeeinheiten Ieisten sollen, als sie bei einem AnschluB von $13 \mathrm{~mm}$ unten und oben leisten könnten.

Alle Arten solcher Fälle anzuführen, ist hier naturgemäß nicht möglich, diese Hinweise dürften indessen für einen einigermaßen umsichtigen Projektanten genügen, um ihn auf den richtigen Weg bei ähnlichen Vorkommnissen zu weisen. Im allgemeinen empfichit sich folgende Regel: Man mache dann, wenn von der tabellenmäßig sich ergebenden Anschlußdimension etwa doppelt so viel Wärmeeinheiten geleistet werden können, als tatsächlich zu leisten sind, einen der Anschlüsse - ob den oberen oder den unteren ist ziemlich indifferent - eine Dimension schwächer, als es die Tabelle angibt.

Selbstverständlich ist es, daß man sehr langen Heizkörperverbindungen nicht die Dimension $13 \mathrm{~mm}$, namentlich in den unteren Geschossen, geben wird.

Schließlich sind noch die Kellerheizkörper zu erwăhnen, welche jetzt häufiger - besonders bei Villen - an die Verteilungsleitung anzuschließen sind. Bei der Dimensionierung der selben spricht meist weniger die Druckhǒhe mit als die Rohrabkühlung. Macht es keine besonders hohen Kosten, so ist es angebracht, die Kellerheizkörper mit besonderer Zu- und Rückleitung zu versehen Diese Leitungen sind dann nach Recknagel $\mathrm{zu}$ berechnen wie die Heizkörper einer Etagenheizung. Ist eine besondere Leitung hingegen aus Gründen zu hohen Kostenaufwandes nicht möglich, so können die Kellerheizkörper auch von der gemeinsamen Verteilungsleitung abgenommen werden. Ihre Anschlüsse sind alsdann so weit zu bemessen, als die besondere Leitung zu ihnen weit sein müßte. Außerdem ist hierbei $\mathrm{zu}$ beachten, daß die horizontalen $\mathrm{Zu}$ - und Rückleitungen, von 
welchen sie abzweigen, naturgemaß nie schwächer, sondern stets mindestens um zwei Dimensionen stärker als der für den Kellerheizkörper erforderliche Anschluß sein müssen. Nähert sich die Zahl der Kellerheizkörper der Anzahl von Heizkörpern einer ganzen Etage, so muß an die Stelle der Tabellen die Berechnung treten.

An Hand des folgenden Beispieles mögen die vorhergehenden Erörterungen năher erlăutert werden.

Die Anlage habe die Stränge I bis V. Unter der Bezeichnung der Stränge sind die horizontalen Entfernungen derselben vom Kessel in Metern verzeichnet. Die Mitte des am ungünstigsten gelegenen Heizkörpers im ErdgeschoB liege 2,8 m über der Mitte des Kessels. Da nur je eine Serie Tabellen für 2,5 und 3,0 m Entfernung zwischen den Mitten des Kessels und des ungünstigsten Heizkörpers vorhanden ist, so wird der Sicherheit halber die erstere gewăhlt.

Strang 1 liegt $8,0 \mathrm{~m}$ vom Kessel entfernt, also ist für die linke Hălfte mit $10,0 \mathrm{~m}$ horizontaler Ausdehnung zu rechnen, wăhrend für die rechte Hälfte und den Kesselanschluß mit einer solchen von $20,0 \mathrm{~m} \mathrm{zu}$ rechnen ist, da Strang $\mathrm{V}$ in einer Entfernung von $18,0 \mathrm{~m}$ vom Kessel liegt. Die Horizontalleitung nach Strang IV, die von der horizontalen Leitung nach Strang V abzweigt, ist, da Strang IV nur 12,0 m vom Kessel abliegt, nach der Tabelle für 15,0 m Horizontalausdehnung zu bestimmen.

Bei der Bestimmung der Leitung nach Strang I ist noch zu beachten, daß die Zuleitung genau den Angaben der Tabelle entspricht, dagegen die Rückleitung eine Dimension schwächer gewählt ist, weil für 15900 WE die Rohrweite $33 \mathrm{~mm}$ und für $24900 \mathrm{WE}$ die Rohrweite $40 \mathrm{~mm}$ fast noch genügen würde.

Über die Bestimmung der Heizkörperanschlüsse der einzelnen Stränge ist kurz noch folgendes zu sagen.
Strang I und II werden für $10 \mathrm{~m}$, Strang III und V für $20 \mathrm{~m}$, Strang IV für $15 \mathrm{~m}$ horizontale Ausdehnung bestimmt.

Die obersten Heizkörper an Strang I erhalten tabellenmäßig Ventile von $13 \mathrm{~mm}$ l. Durchm., ihre Rückleitungen hingegen können, da für die von ihnen zusammen geleisteten Wärmeeinheiten noch immer ein Strang von $13 \mathrm{~mm}$ l. Durchm. genügt, ohne Bedenken eine Lichtweite von $10 \mathrm{~mm}$ erhalten. Aus ganz ähnlichem Grunde erhalten die oberen Anschlüsse der ErdgeschoBHeizkörper an Strang I die Dimensionen 26 bzw. 20, die unteren dagegen nur 20 bzw. $13 \mathrm{~mm}$.

Strang II hat keinen Erdgeschoß-Heizkörper, für diesen Strang erfolgt also die Bestimmung der Rohrweiten nach der rechten Hälfte der Tabelle und zwar wird hierbei die Unterrubrik für 5,0 m Entfernung der Dimensionierung zugrunde gelegt.

Ganz analog erfolgt die Dimensionierung der Stränge III und $V$ nach der Tabelle für $20,0 \mathrm{~m}$ und des Stranges IV nach derjenigen für $15,0 \mathrm{~m}$.

Bei Strang IV werden die Zuleitungen zum I. und II. Obergeschoß hierbei genau nach der Tabelle gewählt, indessen erscheinen sie immerhin so reichlich, daß die entsprechenden Rückleitungen eine Dimension geringer sein können.

Strang $V$ hat im ErdgeschoB keinen Heizkörper. Daher ist für die Bestimmung seiner Rohrweiten wie bei Strang II die rechte Hälfte der zugehörigen Tabelle gewählt. Hierbei tritt der Fall ein, daß beim Übergang von der horizontalen zur vertikalen Leitung eine Reduzierung der Rohrweiten eintritt.

Schließlich sei noch der Kellerheizkörper erwähnt. Sein Anschluß ergab sich rechnungsmäBig als $26 \mathrm{~mm}$. Er kann also ohne Bedenken an die $50 \mathrm{~mm}$ starke horizontale Leitung angehängt werden. 